\title{
Elementos para la construcción social del chedungun a partir del discurso en torno a la lengua de hablantes bilingües de la VIII Región
}

\author{
Patricia Ojeda* \\ Javier Álvarez ${ }^{* *}$ \\ Universidad de Chile, Chile
}

\begin{abstract}
Resumen
Este artículo tiene por objetivo describir e interpretar el discurso de los hablantes bilingües en español y chedungun en el marco de una construcción social de la lengua. Consideramos que realizar un acercamiento a diferentes discursos en circulación sobre el chedungun puede posibilitar la comprensión sobre cuál es el contenido de las actitudes, representaciones sociales y lealtades lingüísticas de los hablantes. Los discursos analizados corresponden al de ancianos, adultos y jóvenes bilingües legitimados en sus comunidades. Este estudio se inscribe en el ámbito de la etnolingüística, es de carácter exploratorio y de enfoque cualitativo. Los datos fueron obtenidos a partir de entrevistas semi-estructuradas, realizadas en el marco de trabajos etnográficos que forman parte de las investigaciones
\end{abstract}

* Para correspondencia, dirigirse a: Patricia Ojeda (pojeda.uchile@gmail.com), Universidad de Chile, Facultad de Filosofía y Humanidades, Programa de Magíster en Lingüística, Av. Capitán Ignacio Carrera Pinto 1025, Ñuñoa, Santiago, Chile. Becaria de la Comisión Nacional de Investigación Científica y Tecnológica de Chile (CONICYT).

** Para correspondencia dirigirse a: Javier Álvarez (j.alvarez.v@hotmail.com), Universidad de Chile, Facultad de Ciencias Sociales, Programa de Magíster en Ciencias Sociales, Ignacio Carrera Pinto 1045, Of.31, tercer piso, Nuñoa, Santiago, Chile. 
Fondecyt Iniciación 11110362 “Caracterización etnolingüística de producción y reproducción de la lengua mapuche rural en comunidades pehuenches de Pitril y Caillaqui, Alto Bío-Bío", y de la tesis de grado de Magíster en Ciencias Sociales, mención Sociología de la Universidad de Chile: "Creencias religiosas y chedungun en las comunidades mapuche en conflicto de la zona de Nahuelbuta".

Palabras clave: construcción social de la lengua, mapudungun y religión, mapudungun y ontología de la lengua.

ELEMENTS FOR THE SOCIAL CONSTRUCTION OF CHEDUNGUN THROUGH METALINGUISTIC DISCOURSES OF BILINGUAL SPEAKERS FROM THE VIII ReGion of CHILE

\begin{abstract}
This paper aims at describing and interpreting the discourse of bilingual speakers of Spanish-Chedungun in the context of an approach to a social construction of the language. An approach to different discourses regarding Chedungun may allow the understanding of the attitudes, social representations and language loyalties of speakers. We analyzed the discourse of bilingual elders, adults and young people recognized in their respective communities. The present qualitative-exploratory study is framed in the field of Ethnolinguistics. The data was gathered through semi-structured interviews, as part of ethnographic field work in the Fondecyt Iniciación 11110362 “Caracterización etnolingüística de producción y reproducción de la lengua mapuche rural en comunidades pehuenches de Pitril y Caillaqui, Alto Bío-Bío", and the Master thesis "Creencias religiosas y chedungun en las comunidades mapuche en conflicto de la zona de Nahuelbuta".
\end{abstract}

Keywords: social construction of language, religion and Mapudungun, the Mapuche language and ontology of language.

Recibido: $15 / 07 / 14 \quad$ Aceptado: $27 / 10 / 14$

\title{
1. INTRODUCCIÓN
}

El estudio de las representaciones sociales, de las actitudes lingüisticas y de las ideologías lingüisticas en torno al mapudungun ha sido abordado en 
el ámbito chileno con el objetivo de desarrollar una perspectiva lingüística que considere las determinaciones históricas y culturales que operan en las lenguas, destacando funciones y contenidos que sobrepasan su mera función comunicativa (Mariano et al. 2010; Lagos 2006, 2010 y 2012; Olate y Henríquez 2010). Desde esta perspectiva, por ejemplo, Chi (2011) describe la forma en que la lengua maya actúa, en algunos casos, como un filtro de comparación social, útil para caracterizar grupos autodefinidos y socialmente reconocidos.

El análisis de las representaciones sociales ha centrado su atención en el proceso de cognición social que subyace en las pautas de comunicación y de comportamiento de los individuos, describiendo la forma en que los grupos y los sujetos configuran la realidad y su modo de estar en ella (Moscovici 1986; Moñivas 1994). Por su parte, el ámbito de las actitudes lingüísticas se ha interesado, principalmente, en las evaluaciones de aprobación o desaprobación que realizan los hablantes o comunidades de hablantes ante un rasgo o una variedad lingüística determinada (Moreno Fernández 2009, Olate y Henríquez 2010). En esta misma línea, aún más recientes son los trabajos que han incorporado el estudio de las ideologías lingüísticas, que examina las creencias implícitas o explícitas sobre dimensiones y usos del lenguaje y su relación con los intereses de determinados grupos sociales (Kroskrity 2010).

En el caso de las lenguas indígenas en Chile,Mariano et al. (2010) y Lagos (2006, 2010 y 2012) han estudiado las representaciones sociales en torno al mapudungun de los mapuches urbanos. Sus resultados señalan que estos conciben esta lengua como una herramienta simbólica útil para la construcción de "un horizonte de sentido respecto de lo que es ser mapuche hoy" (Lagos 2010: 53). Del mismo modo, para Olate y Henríquez (2010), las actitudes lingüísticas cumplen un rol central en la delimitación de comunidades de hablantes y en la difusión de cambios lingüísticos. En su trabajo analizan las actitudes hacia el mapudungun de profesores que trabajan en colegios donde se imparte el Programa de Educación Intercultural Bilingüe en zonas rurales de las regiones VIII y IX, en él constatan una actitud positiva por parte de los profesores hacia la lengua, pues le atribuyen funciones interétnicas, entendiéndola como una vía de conservación del saber ancestral, prevaleciendo este valor simbólico por sobre la funcionalidad comunicativa. Por último, en el ámbito de las ideologías lingüísticas, Lagos, Espinoza y Rojas (2013), examinan la relación entre la ideología de la lengua estándar, los intelectuales mapuches y sus negativas consecuencias en la revitalización del mapudungun.

En cuanto a este trabajo, consideramos que al hacer referencia al estado actual de una lengua minorizada como el chedungun, necesariamente se 
deben tener en cuenta los cambios que se han producido en la estructura global que define y norma las situaciones de contacto lingüístico. Por tanto, el análisis de los discursos en torno a una lengua indígena como el chedungun debe considerar el proceso colonial que enfrentó la sociedad mapuche. La gravitación de ese proceso es tal que, en muchos aspectos, la relación entre las comunidades mapuches y el Estado chileno ha implicado comprenderlo en el marco de la prolongación de la situación colonial que tiene por hito la Pacificación de la Araucanía (Comisión Verdad Histórica y Nuevo Trato con los Pueblos Indígenas 2008).

Así, este trabajo examina el discurso sobre el chedungun -variedad hablada por los pehuenches de la VIII región de Chile y los mapuches de Nahuelbuta (Salas 2006)-, a partir del testimonio de cinco hablantes bilingües (competentes en chedungun y en español) que presentan distintos niveles de bilingüismo y pertenecen a comunidades de las zonas rurales del Alto Biobío (Caillaqui y Pitril) y de la ribera noreste del lago Lleulleu (sector de Rukañanco y Coihueco). Consideramos que analizar el contenido de los distintos discursos que poseen los hablantes en torno a su lengua resulta sustancial para lograr el objetivo que nos hemos trazado: entender las lenguas como una construcción social que se nutre de las creencias, usos y valoraciones que realizan los propios hablantes sobre ellas. En este marco, entendemos que las actitudes, representaciones e ideologías lingüísticas que circulan en las comunidades constituyen aspectos parciales de la construcción social del chedungun. Nuestra propuesta es que para caracterizar esa construcción social se debe situar esos discursos en función de roles y posiciones que los soportan en las comunidades. Es decir, entender la diversidad de discursos sobre el chedungun que resultan de comunidades socioculturalmente desintegradas como consecuencia de la prolongación de la situación colonial que define las relaciones entre sociedad mapuche y la chilena.

Este estudio se inscribe en el ámbito de la lingüística antropológica, es de carácter exploratorio y de enfoque cualitativo. Los datos fueron obtenidos a partir de entrevistas semiestructuradas, realizadas en el marco de trabajos etnográficos que forman parte de las investigaciones Fondecyt Iniciación $11110362^{1}$ y de la tesis de grado de Magíster en Ciencias Sociales, mención Sociología de la Universidad de Chile: "Creencias religiosas y chedungun en las comunidades mapuche en conflicto de la zona de Nahuelbuta". Para

\footnotetext{
Fondecyt Iniciación 11110362 “Caracterización etnolingüística de producción y reproducción de la lengua mapuche rural en comunidades pehuenches de Pitril y Caillaqui, Alto Bío-Bío", dirigida por el Dr. Cristián Lagos.
} 
este trabajo hemos analizado cinco discursos de sujetos hablantes que están validados en su comunidad: una médica $(82)$, tres comuneros $(80,65,44)$, $\mathrm{y}$ un educador tradicional (26).

A continuación, en el segundo apartado, describiremos los conceptos a partir de los cuales se abordarán los discursos de los hablantes, los que comprenden: exposición de los procesos históricos relevantes que explican la diversidad de discursos en las comunidades mapuches; luego, expondremos una aproximación al bilingüismo que contribuya a caracterizar a nuestros entrevistados en esta dimensión. En el tercer apartado referiremos la metodología empleada en este trabajo. En la cuarta sección presentaremos el análisis y los resultados de esta investigación, el que está dividido en los subapartados: a) Bilingüismo: un aspecto de vitalidad lingüística, b) Chedungun y función religiosa y c) Chedungun, lengua y ontología.

\section{ANTECEDENTES}

\subsection{Perduración de la Situación COLONIAL: TRANSFORMACIONES CULTURALES Y LINGÜÍSTICAS EN EL TRÁNSITO DE LA SOCIEDAD MAPUCHE REDUCCIONAL A LA POSTREDUCCIONAL}

En la cultura de las comunidades mapuche del Lleulleu y el Alto Biobío circulan diferentes discursos sobre el chedungun. Esta heterogeneidad se explica por múltiples factores históricos, políticos, culturales y lingüísticos. Entre ellos destacan aquellos que refieren a la cosmología y la religiosidad tradicional. Estas representaciones se nutren de creencias que sobrevivieron a la política estatal de aculturación que sucedió a la pacificación de la Araucanía. Sobreviven en los símbolos y saberes que se vinculan con las creencias religiosas principales como Chao Dios, el newen (fuerza primordial), ngen (espíritus) (Foerster 1995) que se actualizan en ritos religiosos como rogativas y sanaciones chamánicas.

Sin ser residuales, los discursos que vinculan la lengua a dimensiones de las creencias tradicionales religiosas no constituyen un cuerpo articulado de conocimientos compartidos por todos los miembros de la comunidades, menos se trata de un saber integrado. La cultura e ideologías que circulan en la sociedad chilena han penetrado en las comunidades como resultado de su integración forzada al mundo wingka. Esta lógica de integración opera como una fuerza de desintegración sociocultural respecto de los 
sistemas simbólicos mapuche. Actúa, por ejemplo, en la dimensión lingüística, la ideología del monolingüismo, mediante las instituciones del Estado, el sistema educativo y los medios de comunicación. Frente a ella, la representación tradicional del chedungun se puede describir como una ideología lingüística opuesta, que expresa el punto de vista y los intereses de los roles y posiciones tradicionales en las comunidades. De esta manera la construcción social del chedungun es una mirada sincrónica a la disputa y conflicto entre estas ideologías lingüísticas (junto a otras) que revela una correlación de fuerzas entre los distintos saberes y poderes que se posicionan en las comunidades.

Para entender cómo son producidas y recepcionadas dichas ideologías lingüísticas en las comunidades hay que examinar los principales procesos socioculturales que han atravesado. Es decir, examinar lo que se ha llamado resistencia cultural mapuche (Bengoa 1985), que hace posible distinguir, al interior de las comunidades, a los sujetos portadores del saber mapuche y que, en consecuencia, vinculan la ontología y la práctica de su lengua con las creencias tradicionales. En nuestra propuesta consideramos relevante referir el marco colonial en que se han desarrollado las relaciones entre chilenos y mapuche a partir de la ocupación de la Araucanía.

Una vez terminada la ocupación, desplazamiento y derrota militar de la población, en 1883, el Estado chileno condujo el proceso de reducción en comunidades que duró hasta 1929. Paralelamente se va desarrollando la colonización del territorio, de manos de europeos y chilenos. Contrariamente a los cálculos de los políticos chilenos, la sociedad mapuche logró resistir la desintegración social y cultural a la que había sido arrojada por eso proceso colonial mediante la reorganización de sus vínculos económicos, religiosos y lingüísticos. Aparece lo que en la literatura histórica y antropológica se conoce como "sociedad mapuche reduccional". La comunidad mapuche quedó en una situación estructural respecto de la propiedad colonial y la sociedad chilena, que generó las condiciones para mantener grados de autonomía frente a esta. Este rasgo fue destacado en los trabajos etnográficos de finales de la década de 1950 (Faron 1969). Se demostró que frente a la sociedad chilena la cultura mapuche habría mantenido un equilibrio estructural (entre integración y conservación), permitiéndole subsistir. Esta subsistencia logró conformar una estructura cultural diferenciada.

El modo en que se logró fue que las reducciones o comunidades adaptaron la estructura familiar matrilateral. Así, la cesión y recepción de mujeres por vía materna incentivó la endogamia entre colectividades interrelacionadas, manteniendo espacios con relativa autonomía. Igualmente, esta integración familiar permitió la emergencia de lo que Faron (1969) llamó el congregacionismo ritual mapuche. Este sistema de intercambio 
de esposas institucionalizó un sistema de intercambio económico, solidario (mingako) y aseguró la unidad moral de las comunidades, al consagrar el vínculo religioso en la concurrencia a rituales fundamentales:

Dentro del contexto del orden moral la sociedad mapuche vive en mayor aislamiento debido a su situación colonial y es respecto a los valores morales como debe verse la integración continuada. La integridad estructural de la sociedad mapuche es un compuesto de resistencia y adaptación (Faron 1969: 243).

La situación de "equilibrio estructural" que permitió el surgimiento de la sociedad mapuche reduccional se modificó radicalmente con las transformaciones globales del agro chileno a partir de la década de 1960. La reforma agraria y la contrarreforma agraria, con los decretos-ley 701, de 1974 (sobre Fomento Forestal) y 2568/2750 de 1979 (división de tierras y la liquidación de la propiedad comunitaria), generaron el marco jurídico para que el territorio mapuche se anexe completamente a la sociedad chilena, mediante la introducción del capital forestal. Así lo demuestran los estudios más recientes realizados a partir de datos censales (Saavedra 2006). La integración sociocultural de las comunidades respecto al mundo winkga sería, prácticamente, total.

Sin embargo, estos estudios se equivocan al presumir la aculturación total de las comunidades mapuche. Sabemos que la neoliberalización del agro implicó al mismo tiempo su ingreso a la globalización. La masificación de los medios de comunicación, permite el acceso de las comunidades a los instrumentos internacionales de reconocimiento cultural. A partir de la década de 1990, surge una reapropiación y revalorización de las prácticas culturales tradicionales que se habían mantenido latentes en las comunidades más apartadas y entre las generaciones más antiguas. En esa coordenada, espacial y social, pareciera intersecarse vitalidad lingüística y práctica religiosa en figuras que ponen en circulación ese contenido tradicional que se desliza en cada operación religiosa, en la observancia de las creencias y en el uso de la lengua.

En consecuencia, proponemos analizar el discurso de los hablantes, vinculando las representaciones y creencias que comporta esa ideología en su anclaje al contexto sociocultural en que opera ${ }^{2}$. De acuerdo con Foerster (1995), la religiosidad mapuche se enmarca en esquemas de reciprocidad para con los miembros de la comunidad de creyentes y respecto de las figuras

2 Kroskrity llama a este enfoque "análisis ideológico neutral” (2010). 
sagradas. De acuerdo con Durkheim (1982), la distinción sagrado/profano es constitutiva de los sistemas religiosos. Más allá de las representaciones que se asignan a uno $\mathrm{u}$ otro polo, dicha distinción cumple una función social: refuerza lazos entre la comunidad creyente a la vez que distingue/ separa respecto de quienes no participan de las creencias y ritos. Como expondremos, el chedungun es representado, por algunos hablantes, como un don de Chao Ngnechen, y es por lo tanto sagrado, frente al español (wingkadungun) que es considerado profano. El carácter sagrado de la lengua mapuche permite que su manipulación ritual por el ngepin (sacerdote) y la machi (chamán) en el guillatún, tenga eficacia simbólica. La integración de la comunidad de creyentes (a base de esquemas de reciprocidad) es reforzada toda vez que el desarrollo de una liturgia en chedungun, durante el nguillatún, acerca a los participantes (que en su mayoría no son hablantes).

Desde esta perspectiva, sería factible entender que la eficacia ritual del chedungun tiene que ver con la legitimidad de quienes asumen el rol de sacerdotes en el guillatún. Dicha legitimidad está dada, como hemos dicho, en su carácter de hablantes. Esta relación entre palabra y ritual es examinada por Bourdieu (2001). Para que el ritual funcione debe haber una "delegación de la palabra" a quien está legitimado para actuar en nombre de la comunidad cúltica constituida en él.

La eficacia simbólica de las palabras sólo se ejerce en la medida en que quienes la experimentan reconocen que quien la ejerce está autorizado para ejercerla. (...) Reside así totalmente en la convicción de que esa delegación constituye los cimientos mismos del ministerio, esa ficción social, convicción que es mucho más profunda que las propias creencias y misterios que el ministerio profesa y garantiza (Bourdieu 2001).

Asimismo, proponemos complementar la matriz sociocognitiva que subyace al concepto de ideologías lingüisticas, junto con sugerir un diálogo con la propuesta de la epistemología del hablante (Teillier 2013), en cuanto esta postula que la lengua es una construcción social que configura un ámbito de realidad -social- dentro de los límites de las posibilidades de autocomprensión que la constituyen como mundo y cultura. En este sentido, la epistemología del hablante permitiría reconstruir la lengua en contextos sociales complejos, marcados por la modernización capitalista. Igualmente, consideraremos algunos elementos del llamado giro ontológico llevado a cabo recientemente en la antropología cultural. Este enfoque postula la necesidad de profundizar en los procesos de simbolización de la realidad de los grupos indígenas, ir a sus cosmologías donde se articulan símbolos y significados que remiten a su existencia en el mundo y a la relación entre naturaleza y cultura que de ahí se desprende. En ese sentido, los objetos de 
la antropología no serían epistemologías, sino que las ontologías (Viveiros de Castro 2003).

En esa línea hemos comparado nuestros resultados con los del trabajo etnográfico de Magnus Course (2012) en las comunidades lafkenche del lago Budi. Course se inscribe en el giro ontológico y lo utiliza para caracterizar elementos de la cosmología mapuche que dan cuenta de un "perspectivismo ontológico". Uno de esos elementos corresponde a entender que el lenguaje estaría constituido de la misma sustancia de los demás seres de la naturaleza (ñke mapu) y de los seres sagrados (wenumapu). Esa sustancia es el newen (fuerza). Course, señala que los mapuche parecieran ser conscientes de que la palabra (dungu) se encuentra saturada de fuerza (newen) que finalmente distancia cada discurso del control del hablante. La relación entre palabra y autoridad en la sociedad mapuche, estaría dada justamente porque el newen contenido en la palabra del jefe, político (lonko) y religioso (ngepin). En el discurso en torno al chedungun de los hablantes que participan de este trabajo, es posible identificar nociones que dialogan con esta. En el apartado que sigue, referiremos algunos elementos que contribuirán a la caracterización del bilingüismo, considerando un contexto colonial.

\subsection{BILINGÜISMO EN CONTEXTO COLONIAL}

El bilingüismo en América Latina, así como en el resto del mundo, es una situación habitual y no una excepción como ha pretendido representar con las ideologías del Estado monolingüe (Lastra 1992, Lagos 2010). Entre los factores que dan lugar a una situación de lenguas en contacto y el bilingüismo, destacan: los procesos de expansión territorial, los procesos de unificación política y situaciones poscoloniales en comunidades independizadas con hablantes competentes en distintas lenguas (Moreno Fernández 2005).

De acuerdo con Fishman (1988), el bilingüismo es un término acuñado por la psicología para dar cuenta del fenómeno individual del habla, mientras que la diglosia corresponde a la dimensión sociolingüística del fenómeno, siendo posible bilingüismo sin diglosia y viceversa. Enfatizando, precisamente, la dimensión individual del bilingüismo, Weinreich lo define como "la práctica de usar dos lenguas alternativamente" (cit. en Lastra 1992: 172). Esta práctica se manifestaría en tres tipo de hablantes: a) bilingües coordinados, quienes mantienen los sistemas o subsistemas lingüísticos separados, b) bilingües compuestos, aquellos que equiparan los dos signos o subsistemas y, por último, c) los bilingües subordinados, quienes tienden a subordinar un sistema a otro (Lastra 1992). 
Para efectos de este estudio, entenderemos el bilingüismo como una situación social que "designa la coexistencia de dos lenguas en la misma comunidad" (Mounin 1979: 28). Asimismo, incorporaremos los aportes de la teoría de la etnificación a su compresión general. Este enfoque entiende el comportamiento bilingüe como casos de asimetría lingüística en que las prácticas de estos sujetos bilingües tienden a variar de acuerdo con el lugar que estén ocupando en el sistema interétnico (Cardoso de Oliveira 2007). Esta perspectiva resulta pertinente, dado que hemos establecido que comprendemos la situación lingüística del chedungun de las zonas del Alto Biobío y del Lleulleu, y, en particular, el discurso de sus hablantes en torno a su lengua, como la imagen actual de una variedad lingüística que se encuentra vinculada a una comunidad que ha ocupado un lugar de subordinación en un contexto colonial. Así, una característica típica del bilingüismo en situaciones de colonialismo lo representa el hecho de que la lengua dominante tiende a relegar a la lengua dominada a su uso privado (Lagos 2010), tal como ocurre en las comunidades estudiadas.

En la tradición lingüística es posible identificar una perspectiva que considera el bilingüismo o la alternancia de código como una deficiencia. Tal es el caso de los análisis de Bloomfield (cit. en Milroy y Muysken 1995). También en esa línea se encuentra el trabajo de Weinreich realizado en la década de 1950, focalizado en la tipificación estructural, sobre todo en el plano léxico del bilingüismo (Lastra 1992). A conclusiones similares llegaron los desarrollos de la psicología educacional conocida como semilingualism, donde se sostenía que los niños bilingües padecían un déficit comunicativo, en tanto, por su condición, no manejaban ninguna de las dos lenguas completamente y por lo tanto no alcanzaban los procesos cognitivos complejos que les permitiera integrase a los sistemas educativos tradicionales. Como contraparte a estas visiones, Gumperz (cit. en Lastra 2012) sostiene que la alternancia de dos lenguas corresponde a un recurso de expresión del hablante y no una limitación.

Emparentado con el concepto de bilingüismo, figura el de alternancia de códigos. Se define como el uso alternado de dos lenguas en el discurso o dentro de la misma oración sin que se observe influencia de la segunda lengua en las secciones en que se utiliza la primera. Igualmente, Weinrech, en un punto intermedio entre bilingüismo y alternancia de lenguas, identifica una nueva dimensión en los hablantes, la define como una predisposición anímica en que las lenguas se convierten en una causa y un símbolo en sí mismas, esta preferencia también incluye la tendencia a utilizar dicha lengua; a esta preferencia la denomina lealtad lingüística (Lastra 1992). Estos conceptos expuestos serán de utilidad al momento de caracterizar aspectos de los hablantes que contribuyeron en este trabajo que presentaremos a continuación. 


\section{METODOLOGÍA}

\subsection{CARACTERÍsticas DE LAS ZONAS ESTUDiadAS}

Esta investigación se llevó a cabo en las comunas de Alto Biobío y Contulmo, ambas de la octava región. Alto Biobío es una comuna cordillerana de la Provincia de Biobío. Tiene 9.078 habitantes, de los cuales el 71\% se declara mapuche pehuenche $e^{3}$. Se trabajó en las comunidades de Pitril y Caillaqui. La primera se extiende por el cajón del Queuco, desde el Estero Otué por el norte, Estero Boquiamargo y río Turbio por el sur, el río Queuco por el poniente, y las veranadas de Nepún por el oriente. Posee 407 habitantes, de los cuales un $75 \%$ se considera pehuenche. La comunidad de Caillaqui se emplaza en las cercanías de Ralco y cuenta con un total de 637 habitantes, de los que un $85 \%$ se considera de origen pehuenche ${ }^{4}$.

En Contulmo, provincia de Arauco, el trabajo se llevó a cabo en la zona del lago Lleulleu, emplazado en la cordillera de Nahuelbuta. Concentra una alta densidad de población mapuche (2626 personas) que corresponde al $15 \%$ de la población indígena de la provincia de Arauco $^{5}$. La zona del lago Lleu posee una extensión de 670 kilómetros cuadrados. Históricamente se ha identificado a la población mapuche de la zona del Lleulleu como lafkenche, por su cercanía con las comunidades de la costa (Tirúa). En la actualidad muchos rechazan esa identidad, identificándose como mapuche simplemente o mapuche-lleulleuche. El trabajo de campo se realizó en las comunidades de Rukañanco y Calbullanca Nahuelqueo. La primera se emplaza a $26 \mathrm{~km}$ al sur de la comuna de Contulmo, en la ribera noreste del lago Lleulleu. Se encuentra a dos kilómetros de la localidad de Huillinco, donde viven mayoritariamente familias chilenas. El plano básico de la comunidad tiene una extensión de 125 hectáreas (sin contar territorio recuperado a partir de 1998) y viven 22 familias en su interior. La comunidad Calbullanca Nahuelqueo se ubica unos 10 kilómetros al oeste de Rukañanco, en el sector de Coihueco. El lugar se encuentra bastante aislado y se accede por caminos no pavimentados. Es una comunidad joven, se constituyó legalmente el 2013.

\footnotetext{
Encuesta CASEN 2006.

4 En ambas comunidades, la actividad económica está dada, principalmente, por la ganadería y agricultura a pequeña escala, y turismo. Las vías de acceso son de regular calidad, situación que se repite y agrava cada vez más a medida que se avanza por el cajón del Queuco.

5 Ministerio de Planificación y Cooperación, 2001.
} 
Habitan unas 20 personas en su interior, siendo la familia Calbullanca el lof (linaje) principal.

\subsection{SuJETOS PARTicipantes}

La selección de informantes fue intencional, buscando encontrar sujetos con características especificadas previamente. Se procedió por muestreo de tipo bola de nieve, a partir de informantes principales en ambas zonas que ayudaran a localizar a los sujetos que cumplieran las características buscadas. En Alto Biobío los informantes principales fueron directores de escuelas rurales y, en la zona del Lleulleu, el vocero de la comunidad Rukañanco. La distribución de participantes corresponde a tres hablantes pertenecientes a las comunidades de la comuna Alto Biobío y dos de las comunidades de la zona del lago Lleulleu en la comuna de Contulmo.

Los participantes del estudio debían cumplir con el siguiente perfil:

1. Autodefinirse como mapuche, pehuenche o lleulleuche.

2. Hablantes bilingües en chedungun y español.

3. Ocupar roles y posiciones validados por sus comunidades.

\subsection{TÉCNICAS DE RECOLECCIÓN Y PROCESAMIENTO DE LOS DATOS}

Se utilizó un enfoque cualitativo y una aproximación etnográfica (seis estadías en terreno de tres a cinco días en las comunidades seleccionadas). Se aplicaron entrevistas semiestructuradas en las casas de los entrevistados o en fogones contiguos a sus hogares, primando una situación comunicativa formal. Las preguntas fueron formuladas en español y las respuestas de los entrevistados fueron expresadas en español, alternancia entre españolchedungun y segmentos completamente en chedungun. Se solicitó a los sujetos entrevistados referirse a los siguientes tópicos:

a) qué significa hablar y comunicarse en chedungun,

b) situaciones en que resulta deseable hablar chedungun,

c) situación de la lengua entre los jóvenes de la comunidad,

d) relación entre ser pewenche/mapuche y hablar chedungun.

Los datos fueron procesados con asistencia del software Atlas.ti, utilizando técnica de codificación de acuerdo a la de la teoría fundamentada: 
codificación abierta, axial y selectiva. Generación de categorías centrales y unidades de sentido.

\section{RESULTADOS}

\subsection{BILINGÜISMO: UN ASPECTO DE LA VITALIDAD LINGÜÍSTICA}

La situación lingüística actual del mapudungun en Chile es de un progresivo desplazamiento funcional en favor del español (Lagos 2005 y 2012; Zúñiga 2007). En la zona del Alto Biobío y del Lleulleu es el español la variedad que domina en los espacios públicos (escuela, municipalidad, biblioteca), perdurando la lengua nativa, principalmente en el ámbito privado -tal como suele ocurrir en contextos de fricción étnica-. La subsistencia del chedungun, en muchos casos, termina siendo una decisión familiar en que los padres establecen si transmitir o no la lengua a las nuevas generaciones. De acuerdo con el testimonio de los sujetos que participaron de la investigación, en esta decisión influyen tanto su propia trayectoria como hablantes de lengua indígena -marcada por la discriminación-, como la escasa presencia de los espacios de dominio de uso del chedungun que, en términos prácticos, reduce las posibilidades efectivas de emplearlo con funcionalidad comunicativa.

Teniendo en cuenta el estado actual de esta variedad, resulta pertinente interpretar la cualidad de bilingües de nuestros entrevistados como un tipo de lealtad lingüistica que contribuye a caracterizar de forma complementaria los datos cuantitativos sobre vitalidad que circulan en la actualidad (Lagos 2005; Zúñiga 2007; Gundermann, Canihuan, Clavería y Faúndez 2008) que tienden a excluir la perspectiva en torno a la lengua de los usuarios. En este sentido, coincidimos con las observaciones expuestas por Teillier (2013), quien critica el predominio de enfoques cuantitativos para medir vitalidad lingüística. Como contraparte, propone incorporar una dimensión cualitativa que se busque superar la dicotomía academia-comunidad estudiada, para esto, sugiere la reconstrucción de una "epistemología del hablante" que explore los puntos de vista de los sujetos que conforman las comunidades lingüísticas a fin de establecer un diálogo entre estos y su realidad socio-cultural. Adscribiendo a estos planteamientos, ahondaremos en las valoraciones y funciones que los hablantes bilingües en chedungun y español de la zona del Alto Biobío y Lleulleu atribuyen a la lengua nativa para inscribirlos en una construcción social del chedungun. 
Los sujetos que participaron en este trabajo presentaban distintos niveles de bilingüismo: entrevistado 1 (E1): bilingüe coordinado, L1 chedungun; entrevistado 2 (E2): bilingüe compuesto, L1 chedungun; entrevistado 3 (E3): bilingüe pasivo, L1 español; entrevistada 4 (E4): bilingüe subordinado, L1 chedungun; entrevistado 5 (E5): bilingüe subordinado, L1 chedungun. Por su parte, los entrevistados de mayor edad (E2 y E5) presentaron una alta frecuencia de alternancia de código:

"Mucha gente nueva wekeche cambia letuykimtulaydew chedungun (la gente joven está cambiando, ya no saben su idioma), entonces así estamos perdiendo como, como le dijera porcionada de mapuche" (E2).

“chedungun le voy a decir kiñeke che, kiñekengvtram, kiñekedungu, kiñeke (alguna spersonas, algunas conversaciones, algunos asuntos, algunos $)^{6}$ tal como el objeto, no es igual al que hablan los que viven cerca de los lafquenche" (E5).

La motivación de la alternancia de código español/chedungun y los segmentos expresados completamente en chedungun deben considerar las restricciones que la situación comunicativa de entrevista implica, en que el objeto y tópico conversacional es, precisamente, la lengua. Sin embargo, al margen de este contexto, es innegable que este tipo de alternancia de código refleja, en los mismos sujetos, los efectos de la prolongación de las relaciones coloniales en las comunidades mapuches. Este efecto desintegrador también puede ser leído como una valiosa muestra de lealtad lingüistica en un contexto de claro desplazamiento y baja vitalidad de la lengua, siendo un dato que contribuye a conformar la epistemología de los hablantes. Esta alternancia de códigos lejos de ser una deficiencia o una interferencia (Lastra 1992), en el caso de los mapuches de la VIII región simboliza una resistencia a la aculturación impuesta por el mundo winkga ${ }^{7}$. Esta perspectiva se confirma, además, explícitamente con sus testimonios:

6 Agradecimientos a Antonio Chihuaicura, quien tradujo los segmentos en chedungun.

7 Con esto no queremos establecer, necesariamente, que la lealtad hacia el grupo sea más alta entre quienes mantienen su lengua en perjuicio de quienes no. En este punto, concordamos con Fishman (1988), cuando señala que "parece ser que la lealtad al grupo puede ser similarmente (si no más) ubicua y continuar tanto con el mantenimiento lingüístico como sin él" (Fishman 1988: 151). Tal es el caso de las agrupaciones de mapuches urbanos que habitan en la Región Metropolitana y que participan activamente de organizaciones que promueven la identidad étnica sin ser necesariamente hablantes (Lagos 2012). 
“iñchechedunguken, vychekewvneluenewtachaw dios, feyniekefiñ, che amun, pen che, dungukentañikewvn, nvtramkaken chedungun meu, komchemyewvn, chem, chemamon, akuntameu, amonpiken, chedungun mu miawken, wingkadungun newetopakelan" (E5)

[Yo suelo hablar en chedungun, el Chaw Ddios me dio la lengua de la gente, eso es lo que tengo, donde voy, donde veo a las personas, suelo hablar mi lengua, suelo conversar en chedungun, todo los obsequios, que, donde voy, de donde vengo, voy suelo decir, ando con chedungun, el wingkadungun no lo hablo mucho.]

Como elemento constitutivo de esta lealtad figura la relación que establecen algunos hablantes en entender el chedungun como un regalo de Chao dios (E5) o como una enseñanza que proviene de la naturaleza (E1). Esta visión coincide con los datos proporcionados por Olate et al. (2013), quienes examinan la alternancia de códigos en una comunidad de la zona lafkenche; los resultados señalan que los eventos comunicativos "donde más se habla la lengua mapuche son de carácter ceremonial: machitun con $60 \%$ y ngillatun con 80\%; además, el palin con 60\%" (Olate et al. 2013: 272). Información que se corrobora con lo expresado por nuestros entrevistados: "si ese en chedungun se llama, en la rogativa, el ngillatun se llama ngillanmaun de los mapuche. Ahí tenemos que orar los puros mapuche" (E4), configurándose como una dimensión central en la construcción social de la lengua. Nos referiremos en extenso a esta noción en el próximo apartado.

\subsection{FunCIÓN RELIGIOSA DEL CHEDUNGUN}

En el discurso de los hablantes de la zona del Alto Biobío y de la ribera del lago Lleulleu se advierte una relación entre la conservación de las creencias mapuches y el mantenimiento del chedungun; es por este motivo que analizamos los discursos en torno a la lengua nativa que refieren a este vínculo. Esta asociación configura, junto con otros elementos, la construcción social del chedungun que se ha propuesto describir en este trabajo. Para analizar estas representaciones tradicionales, referiremos, brevemente, su estado en un escenario que ha sido descrito como de aculturación sistemática (Saavedra 2006) y de pérdida de vitalidad de la lengua vernácula (Zúñiga 2007, Salas 2006, Lagos 2012) producto de la perduración de la situación colonial en las relaciones entre sociedad chilena y comunidades.

Aunque resulta apropiado hablar de desintegración sociocultural de las comunidades (respecto de lo que se ha llamado sociedad mapuche reduccional) la idea de aculturación puede resultar superficial, dado que no considera los ajustes y adaptaciones de la cultura y lengua mapuche respecto 
de la cultura wingka. De acuerdo con Bengoa (1985), desde la reducción de la población mapuche en comunidades existiría una cultura de resistencia, en la que la cultura mapuche se habría rigidizado y ritualizado como estrategia para subsistir a la integración forzada por la sociedad chilena.

A pesar de la cultura de resistencia, parece indudable que las comunidades han experimentado un proceso de cristianización y castellanización de su población, producto de su integración a los esquemas productivos nacionales y la migración a las ciudades. Sin embargo, el awingkamiento de las comunidades ha sido resistido por un sector que conserva su cultura y lengua tradicionales. En las comunidades, esto ha permitido la reapropiación y revalorización de esas prácticas tradicionales. En ese contexto, los más ancianos, quienes ocupan roles y posiciones asociadas a la cultura y religiosidad mapuche, adquieren un estatus de sostenedores de la cultura:

Que me salgan algunas palabras mayores digamos en esta parte. Y me van a decir "tú de a dónde saliste si estai recién creciendo y te falta mucho para conocer". Claro, a mí me falta mucho pa' conocer. Yo no puedo pasar a llevar a un anciano que quizás es mucho más que yo (E1).

En relación con esto, cabe mencionar que los pueblos amerindios que experimentaron situaciones de colonización presentan, con el advenimiento de la globalización, una actitud de revalorización de sus prácticas culturales tradicionales, sobre todo, respecto de sus lenguas vernáculas. Las tribus yokut y mono en Estados Unidos (Kroskrity 2013) han presenciado una estrategia de reapropiación de sus narrativas orales, de carácter ritual, que combina el rescate de sus manifestaciones latentes, así como de las recolecciones etnográficas de principios del siglo XX. Eso se traduce en un mayor estatus de los ancianos, quienes aún hablan la lengua vernácula y conocen las narrativas tradicionales. Entre los mapuche, la revaloración de la cultura y en particular la lengua, ha significado un mayor estatus asociado a los roles tradicionales, especialmente chamanes, médicos y autoridades locales. Para los más jóvenes en las comunidades ese sector tradicional asume la condición de kimche (personas sabias ${ }^{8}$ ), cuyo indicador más claro es la cualidad de hablante de la lengua: "el chedungun se habla, a través de... tienen que conocer el... el kimün o la sabiduría que a nosotros nos dejaron, porque para hablar chedungun uno tiene que tener conocimiento, un consejo o alguna historia" (E1).

8 Kimún: conocimiento, sabiduría; kimche: persona sabia. 
Asimismo, quienes son hablantes tienen la cualidad de ser especialistas de la lengua, poseedores de un capital simbólico y religioso reconocido en las comunidades indígenas. Montemayor (2013) ha descrito este proceso de especialización lingüística en distintos grupos indígenas de América Latina en el que la lengua deviene en: "un arte de composición, [que] tiene funciones precisas, en particular la de conservar conocimientos ancestrales a través de cantos, rezos, conjuros, discursos o relatos" (Montemayor 2013: 6).

En el mapudungun, por ejemplo, esa especialización y modalidad compositiva se manifiesta en la circulación de géneros discursivos orales con características estables, como los epew: relatos ficticios, generalmente, protagonizados por animales, cuya objetivo es didáctico o de entretención: "Aquí pasaba lo mismo. Aunque no había tráfico ni nada, igual los papás elegían en las tardes enseñarnos, contarnos epew por ejemplo. A esa hora mi papá me hacía esa... era como una clase así" (E1).

Dado que en el pensamiento tradicional mapuche el chedungun es un don de Dios, es parte de la creación sagrada, junto a la vestimenta, el alimento, los animales y demás seres que habitan la naturaleza (ñuke mapu): "chedungun les será entregado a ustedes, así tendrán conocimiento de chawngvnechen, como han sido dejados, tendrán acceso al conocimiento por el chedungun" (E2).

De esta manera, la palabra (dungu) es concebida dentro del orden cosmológico como el hablar de la tierra (mapu dungu) y hablar de la persona (che dungu). El orden cosmológico, como creación divina es sagrado. La lengua, como parte de ese orden, también es sagrada. En este sentido, la entrevistada E4, médica (lawentuchafe) de 80 años señaló que a ella le gustaba hablar su lengua con sus amigos y familia porque esa es la lengua que Dios nos dejó y que hablarla es agrado en Dios, justamente porque la vinculación con las representaciones sagradas no puede sino producirse en chedungun. El castellano, wingkadungun, es representado como profano, carente de potencial semiótico para vincular al mapuche con los seres sagrados en los que cree.

Siguiendo la tradición mapuche, ella utiliza el espacio onírico del sueño (реuma) para obtener el lawen (conocimiento etnobotánico). En su peuma aparece una machi muy hermosa que le señala qué problema médico tiene o tendrá algún ser querido, qué hierbas utilizar y dónde encontrarlas. Esa machi, dice la médica, le habla en chedungun. Si ella no fuera hablante sería imposible la experiencia en el peuma. Lo mismo en sentido contrario; cuando le reza a Chao Ngnechen y sus espíritus en la tierra (ngen) ella tiene el don de ser escuchada, y eventualmente favorecida, porque sus plegarias son en chedungun. A diferencia de la gran mayoría de su comunidad, que 
no es hablante, ella remarca su newen (poder) en la representación de que "Chao dios no lo recibe en wingkadungun"(E4).

La representación religiosa del chedungun que circula en las comunidades tiene una dimensión activa, que tiene efectos sobre la comunidad de creyentes. Está sancionado que quien oficia en el guillatún debe ser un ngenpin ('dueño de la palabra'), normalmente un lonko o un machi. Para la comunidad de creyentes constituida en ese ritual de fertilidad (rogativa) los aspectos discursivos de la liturgia (la petición a Chao Ngnechen para que asegure buenas cosechas ${ }^{10}$ ) deben ser ejecutados en chedungun. Una liturgia en castellano corre el riesgo de profanar las figuras sagradas llamadas a presentarse en el rito. En los relatos de nuestros entrevistados de la zona del lago Lleulleu, la experiencia numinosa, los aspectos mágicos e inefables de la creencia en el guillatún se produce cuando Chao Ngenechen envía los pulunkos ('mensajeros') que se comunican con la machi. El rol de dungu machife, (traductora de la machi) expone la voluntad del Chao Dios al traducir para los participantes no hablantes el mensaje recibido por la machi. Ocurre, entonces, que la manipulación ritual del chedungun tiene eficacia simbólica; entre los participantes, la mayoría no hablantes, el chedungun actualiza lo sagrado, aparece como un instrumento mágico por el cual los seres divinos manifiestan su voluntad. Incluso E4, al comienzo de la entrevista planteó el inconveniente de conversar con un wingka acerca del chedungun. Ella advirtió que, por ser hija de una machi se le había transmitido un poder, en este caso del lawen. Señaló, entonces, que se limitaría al mínimo a hablarnos en chedungun, y lo haría mayormente en wingkadungun " $(. .$. porque mi poder mío no es bueno que le vaya entrenado por una y otra parte su mapudungun" (E4).

En sus relatos, los jóvenes del Lleulleu que participan del guillatún se autoexcluyen de los roles importantes en el ritual. Asumen el papel de kuriche ('kuri', negro) que se constituye en una especie de coro, en los márgenes del rehue (lugar dónde se efectúa e guillatún) y siguen las instrucciones del Ngenpin y de la machi. Dada la importancia de la rogativa para asegurar buenas cosechas, no quieren profanar con el castellano a los pulonkos que

\footnotetext{
9 En la tradición religiosa mapuche la carrera chamánica se inicia por la presencia temprana (en la infancia) de signos que indican que tiene el don. El primero es el peuma, tener sueños reveladores y el perimontun (visión reveladora). El segundo es la exposición a la enfermedad, sobre todo entre las machi que en su combate al mal que enferma a las personas, se exponen a las "fuerzas del mal". De ahí que una machi pueda, eventualmente, hacer el mal en la forma de brujería (Foerster 1995).

10 En la zona del Alto Biobío se le pide a Antürey, Millarey y Küyenmay (E1).
} 
son llamados a la ceremonia para que lleven el mensaje a Dios. Los pulonkos solo escucharían en chedungun.

En nuestra investigación aparece un discurso complementario a la idea de que el chedungun es un don de Chao Ngnechen. Es el caso del entrevistado E1, un joven educado en el pensamiento tradicional-religioso mapuche (no presenta la trayectoria de aculturación por conversión al cristianismo). Es bilingüe competente en chedungun y español, su primera lengua es chedungun. Su representación de la lengua postula el origen onomatopéyico $^{11}$ de la lengua mapuche. Desplazamiento desde el don Chao Dios a la ñuke mapu que aporta los sonidos y expresiones primordiales de esa lengua. Esta perspectiva viene a confluir con una aparente tendencia a en las lenguas indoamericanas de adscribir a la teoría onomatopéyica del lenguaje (Unesco 1990, Montemayor 2013, Sierra 2010). En el caso del mapudungun, destaca el análisis de Navarro (2014), quien realiza un examen del léxico etnobiológico de la lengua mapuche en la región patagónica argentina, confirmando una importante presencia de léxico en que es posible distinguir motivaciones en el plano del significante. Sin embargo, lo interesante, desde el punto de vista de la antropología lingüística, es que la creencia en el origen onomatopéyico del chedungun muestra una continuidad con la concepción tradicional de la lengua. Así, E1 señala que el chedungun es una enseñanza de la naturaleza. Su discurso expresa un tránsito en la representación del origen de la lengua que va del padre (Chao) que crea y entrega todo a la madre (ñke). Este entrevistado teoriza respecto de un estadio originario en que las personas, colocadas en estado de naturaleza, como animales, empiezan a relacionarse con ella y a nombrarla:

Si eran como animales no más. Uno recogía comida pa’ acá. El otro iba pa' allá. Era como que... como unos animalitos que buscaban comida sobre la tierra, lo que nos dejaba no más. Entonces, ellos empezaron a

11 Cabe señalar que la teoría onomatopéyica del lenguaje figura como un antecedente importante de la tradición lingüística, considerada por la historiografía como un debate primitivo de la Grecia Antigua entre el convencionalismo y naturalismo (Robins 1980, Mounin 1968). Un autor emblemático en la configuración de la lingüística moderna que retoma esta discusión es el propio Ferdinand de Saussure, estableciendo que la arbitrariedad del signo y su carácter no motivado es una propiedad central del signo lingüístico (2012 [1916]). En cambio, Sapir, contemporáneo de De Saussure, se refiere al origen onomatopéyico como un nivel legítimo de estudio. Indica que en las lenguas europeas como el inglés o el alemán abunda un léxico proveniente de sonidos de la naturaleza, característica que no tendrían las lenguas nativas de Estados Unidos. Posteriormente, esta afirmación será desmentida (Navarro 2014). 
observar la naturaleza, identificaron algunas cosas, ellos lo nombraron. Como que ahí surgió algunas palabras, fue surgiendo el chedungun (E1).

En su relato, además, nos proporciona el siguiente ejemplo:

Por ejemplo, camino. En chedungun se dice rüpü, pero rü... rüpü. Entonces cuando yo digo rü. La palabra viene por ejemplo, uno cuando camina emite un sonido (onomatopeya de caminar sobre camino de tierra). Ahí tienes rüpü. Entonces son palabras que vienen de la naturaleza, porque son sonidos que emite el hombre cuando camina sobre la tierra (E1).

A modo de síntesis, es posible afirmar que la función religiosa que los hablantes proyectan sobre el chedungun, junto con constituir un factor de mantenimiento de la lengua, contribuye a perfilar una ontología lingüística que sobrepasa la mera función comunicativa o instrumental -visión propia del pensamiento occidental- ofreciendo, de este modo, elementos útiles para comprender la relación entre los miembros de esta comunidad y el chedungun y, de paso, ampliar los horizontes a partir de los cuales se conciben las lenguas. Esta dimensión ontológica será desarrollada en el siguiente apartado.

\subsection{Chedungun, Lengua y OnTOlogía}

La preocupación por la ontología que comportan las distintas cosmologías en el mundo ha sido introducida por el antropólogo brasileño Eduardo Viveiros de Castro. En su análisis de las culturas amazónicas, el autor descubre que las diferencias con el pensamiento occidental no solo son epistemológicas (relativas a como se conoce la realidad), sino que también involucran una concepción distinta respecto del ser (ontología) de la naturaleza. En lugar de la distinción entre sujeto y objeto (hombre y naturaleza) de la cultura occidental, los miembros de las comunidades amazónicas conciben un mundo compuesto por múltiples subjetividades humanas. Viveiro de Castro denomina a este giro ontológico perspectivismo:

la condición original común entre animales y humanos no es la animalidad, sino la humanidad. En la gran división mítica no es tanto la cultura la que se aleja de la naturaleza como esta que se aparta de aquella (Viveiros de Castro 2003: 204). 
El perspectivismo ha mostrado su pertinencia en el análisis de distintas cosmologías amerindias que comparten nociones ontológicas distintas de las occidentales. En el caso mapuche, el trabajo etnográfico de Magnus Course (2012) aplica este enfoque en comunidades lafkenche del lago Budi. Su trabajo presta especial atención, justamente, a la concepción mapuche de su lengua desde la cual, a su juicio, se evidencia una ontología perspectivista. Course analiza distintas dimensiones de la lengua mapuche a partir de las cuales se desprenden diferencias ontológicas respecto de la lengua y cultura occidentales.

Se ubica la lengua cosmológicamente como parte de la creación sagrada. Vimos también que para los mapuche de ambas zonas la palabra no es una atributo exclusivo de la persona, sino que se manifiesta en distintos seres de la naturaleza y del wenumapu (espacio de los seres sagrados). En la cosmología religiosa mapuche la lengua no es un instrumento que permite enunciar la naturaleza sino un estado de dialogo, en ambos sentidos, con ella. Esta concepción contradice las ideologías lingüísticas dominantes en occidente que representan el lenguaje como un instrumento de comunicación de los hablantes ${ }^{12}$. Una cascada, un zorro o una simple piedra son portadores de la palabra:

nosotros, la palabra de nosotros estamos en mapu, en mapudungun. No es chileno, porque decir mapu, porque uno nació de la tierra, por eso el mapuche se llama mapuche porque nació como nace un árbol así nació uno en la tierra (E4).

El chedungun, en este caso, expresaría la inversión del antropocentrismo occidental: el logos está en la naturaleza no en la cultura. Extiende la humanidad a la naturaleza. Course señala que aquello configura los elementos perspectivistas de la cosmología mapuche. El newen sería un proceso emergente por medio del cual distintas entidades llegan-a-ser (Course 2012). Para Course, la palabra (dungu) está constituida de newen. De ahí que, para el mapuche, el intercambio lingüístico va más allá de lo que los individuos quieren decir. La palabra conecta con el newen de la persona, su intención y bondad ocultas que de alguna manera se reflejan en el poder de sus palabras.

La idea de que la palabra posee un exceso de fuerza significa para Course que la autoridad, en la sociedad mapuche, se constituye en la relación de

12 La sobredeterminación de la realidad en la lengua no es percibida por la conciencia occidental ni las ideologías lingüísticas. Según el sentido común, el lenguaje es transparente, vehiculiza la intención del hablante. Pero la realidad del lenguaje es más obtusa. 
los individuos con la palabra. Relación que se manifiesta en el ritual. Dicha afirmación se vincula con los datos recolectados en esta investigación.

Por último, cabe señalar que asumir que existe y circula, a pesar de la desintegración sociocultural, un pensamiento ontológico entre los mapuche tiene varias implicancias. Es posible pensar que desde esa matriz el presente cosmológico del mapuche sea uno en el que la tierra y la lengua se extinguen inevitablemente. En los testimonios analizados subyace un pesimismo, fatalidad respecto del destino de la lengua. En consecuencia, no está garantizada en ese núcleo tradicional una fuerza para oponer a la pérdida de vitalidad, como supone la teoría de la etno-vitalidad de la lengua (Giles et al., cit. en Teillier 2013). Para hallar núcleos de resistencia lingüística, nos parece, se debe examinar, necesariamente, otras concepciones circulantes sobre la lengua, por ejemplo, la reapropiación del pensamiento tradicional por parte de los mapuches urbanos que ven en este una estrategia política útil para la defensa conjunta del territorio y la lengua.

\section{CONCLUSIONES}

En este trabajo hemos propuesto el concepto de construcción social de la lengua para describir la relación entre las múltiples representaciones sobre el chedungun que circulan en las comunidades mapuche. El concepto permite relacionar esas representaciones a las posiciones y roles en las comunidades, de forma que se revelan como ideologías lingüísticas y también como inscripciones en la religión y cosmología mapuche. Por otro lado, la perspectiva acerca de la prolongación de la situación colonial en las relaciones entre sociedad chilena y comunidades admite historizar esas representaciones sobre el chedungun en términos de los efectos de esa relación. Así, por ejemplo, tanto la presencia del bilingüismo, la subordinación al español, como las representaciones religiosas acerca del chedungun son una expresión de la fuerza desintegradora que suponen las relaciones coloniales.

El contenido de dichas representaciones revela una profusa red de significados y símbolos asociados a la lengua mapuche. Esa subordinación del castellano respecto del chedungun resulta paradójica habida cuenta del desplazamiento funcional y reducción de sus dominios de uso. La paradoja es aún mayor dado que la pérdida de vitalidad de la lengua implica para los pocos hablantes un reconocimiento (kimche), un capital simbólico en 
las comunidades. Se produce, entonces, que la manipulación ritual del chedungun tiene eficacia simbólica: acerca a las participantes a lo sagrado, a la naturaleza y a los ancestros.

Estas representaciones del chedungun revelan, además, una dimensión más profunda, de orden cosmológico, manifestando elementos de lo que ha sido llamado ontología perspectivista. De acuerdo con los miembros de las comunidades, la palabra (dungu) pareciera ser común a los seres naturales y sobrenaturales distribuidos en el espacio natural y mítico. Esta unidad lingüística de los seres disuelve la distinción sujeto-objeto (cultura y naturaleza) en cuanto los últimos (animales, plantas ríos, etc.) son sujetos de palabra (mapu-dungu). Múltiples hablantes suponen múltiples puntos de vista; no hay en ese marco de referencia la idea de una naturaleza común, estable, pasiva como la que se constituye en el pensamiento occidental.

Creemos que las representaciones religiosas y los elementos perspectivistas contenidos en la construcción social del chedungun pueden resultar útiles para distintos campos de investigación lingüística y antropológica. Primeramente, consideramos que el estudio de la vitalidad de esa lengua debe integrar otras dimensiones, tales como el aspecto religioso y cosmológico de sus representaciones, porque estas le dan una fuerza de resistencia particular como supone la teoría de la etnovitalidad de la lengua (Giles et al., cit. en Teillier 2013).

Por último, estimamos que para hallar núcleos de resistencia lingüística, resultaría necesario ampliar el repertorio de sujetos representativos de las distintas perspectivas ideológicas que configuran la sociedad mapuche en la actualidad, es decir, caracterizar íntegramente cómo se está construyendo socialmente la lengua mapuche en las comunidades. Para ello, se deben examinar otros relatos sobre la lengua, pues este estudio examinó, principalmente, el discurso en torno al chedungun de sujetos pertenecientes a comunidades rurales que mantienen las creencias religiosas mapuches tradicionales.

\section{REFERENCIAS BIBLIOGRÁFICAS}

Bailey, Benjamin. 2007. Heteroglossia and boundaries. En Monica Heller (ed.). Bilingualism: A Social Approach, pp. 257-274. Londres: Palgrave.

BengoA, José. 1985. Historia del pueblo mapuche (Siglo XIX y XX). Santiago: Sur. 2005. Memoria, oralidad, escritura. Conferencia inaugural del año académico 2005 de la Escuela de Antropología de la Universidad Academia de Humanismo Cristiano. 
Bourdieu, Pierre. 2001. ¿Qué significa hablar? Economía de los intercambios lingüísticos. Madrid: Akal.

Cardoso de Oliveira, Roberto. 2007. Etnicidad y estructura social. México, D.F.: Universidad Autónoma Metropolitana.

Chi, Hilario. 2011. La vitalidad del maayat'aan. Estudio etnográfico de la comunicación intergeneracional de los mayas de Naranjal Poniente. La Paz: Plural Editores.

Comisión Verdad Histórica y Nuevo Trato con los Pueblos Indígenas. 2008. Informe de la Comisión Verdad Histórica y Nuevo Trato con los Pueblos Indígenas. Santiago: Comisionado Presidencial para Asuntos Indígenas.

Course, Magnus. 2012. The birth of the word. Language, force, and Mapuche ritual authority. HAU: Journal of Ethnographic Theory 2 (1): 1-26.

de Saussure, Ferdinand. 2012 [1916]. Curso de lingüística general. Buenos Aires: Losada.

Durkheim, Émile. 1982. Las formas elementales de la vida religiosa. Madrid: Akal editor.

FAron, Louis. 1969. Los mapuche: su estructura social. México, D. F.: Instituto Indigenista Interamericano.

Fishman, Joshua. 1988. Sociología del lenguaje. Madrid: Cátedra.

Foerster, Rolf. 1995. Introducción a la religiosidad mapuche. Santiago: Universitaria.

Gundermann, Hans, Jacqueline Canihuan, Alejandro Clavería y César Faúndez. 2008. Perfil sociolingüístico de comunidades mapuches de la VIII, IX y X Región. Santiago: Universidad Tecnológica Metropolitana.

Kroskrity, Paul V. 2010. Language ideologies - Evolving perspectives. En Jürgen Jaspers, Jan-Ola Östman y Jef Verschueren (eds.). Society and language use, pp. 192-211. Amsterdam: John Benjamins.

2013. Discursive Discriminations in the Representation of Western Mono and Yokuts Stories. Journal of Folklore Research 50: 145-174.

Lagos, Cristián. 2005. La vitalidad lingüística del mapudungún en Santiago de Chile. Revista Werken 6: 23-38.

2006. Mapudungun en Santiago de Chile: vitalidad, lealtad y actitudes lingüísticas. Lenguas Modernas 31: 97-117.

2010. El mapudungún de Santiago de Chile. Caracterización de la competencia, vitalidad y representaciones sociales en torno a la lengua mapuche en la ciudad. Tesis para optar al grado de Doctor en Filología Hispánica, Universidad de Valladolid.

2012. El mapudungún en Santiago de Chile: vitalidad y representaciones sociales en los mapuches urbanos. Revista de Lingüística Teórica y Aplicada 50 (1): 161-190.

Lagos, Cristián, Cristián Oyarzo, Héctor Mariano, Daniela Molina y Felipe Hasler. 2009. Perfil etno y sociolingüístico del mapudungún en Santiago de Chile. Lenguas Modernas 34: 117-137.

Lagos, Cristián, Marco Espinoza y Darío Rojas. 2013. Mapudungun according to its speakers: Mapuche intellectuals and the influence of standard language ideology. Current Issues in Language Planning (CILP) Vol 14, $\mathrm{N}^{\circ}$ 3: 1-16.

Lastra, Yolanda. 1992. Sociolingüistica para hispanoamericanos. Una introducción. México: El Colegio de México.

Maldonado-Torres, Nelson. 2007. Sobre la colonialidad del ser: contribuciones al desarrollo de un concepto. Bogotá: Siglo del Hombre Editores.

Mariano, Héctor, Daniela Molina, Cristián Oyarzo, Cristián Lagos y Felipe Hasler. 2010. ¿Mongeley kam mongelelay chi mapudungun waria mew? Vitalidad y representación social del mapudungún en Santiago de Chile. Romanitas 4 (2): 42-53.

Milroy, Lesley y Peter Muysken. 1995. One speaker, two languages: cross-disciplinary perspectives on code-switching. Cambridge: Cambridge University Press. 
Montemayor, Carlos. 2013. Arte y plegaria en las lenguas indígenas de México. México, D. F.: Fondo de Cultura Económica.

MoÑivas, Agustín. 1994. Epistemología y representaciones sociales: concepto y teoría. Revista de Psicología General y Aplicada 47: 409-419.

Moreno Fernández, Francisco. 2005. Principios de sociolingüística y sociología del lenguaje. Barcelona: Ariel.

2009. La lengua española en su geografia. Madrid: Arco/Libros.

Mounin, Georges. 1968. Historia de la lingüistica: desde los orígenes al siglo XX. Madrid: Gredos.

1979. Diccionario de lingüistica. Barcelona: Labor.

Moscovici, Serge. 1986. Psicología social. Barcelona: Paidós.

Navarro, Herminia. 2014. El léxico etnobiológico en lengua mapuche. Cuadernos de Lingüística Hispánica 23: 13-28.

Olate, Aldo y Marisol Henríquez. 2010. Actitudes lingüísticas de profesores mapuche de Educación Básica: vigencia y enseñanza del mapudungun en el contexto educativo. Literatura y Lingüística 22: 103-116.

Olate, Aldo, Paula Alonqueo y Jacqueline Caniguan. 2013. Interactividad lingüística castellano/mapudungun de una comunidad rural bilingüe. Alpha 37: 265-284.

Pfeiler, Barbara. 1988. Yucatán: el uso de dos lenguas en contacto. Estudios de Cultura Maya XVII: 423-445.

Robins, Robert H. 1980. Breve historia de la lingüistica. Madrid: Paraninfo.

SAAVEDRA, AlejANDRo. 2006. Las transformaciones de la población mapuche en el siglo XX. Santiago: Grupo de Estudios Agropecuarios.

Salas, Adalberto. 2006. El mapuche o araucano. Santiago: Centro de Estudios Públicos.

Sierra, CALIP. 2010. Quechuapaonomatopeyankuna. Lima: AIDIA.

Teillier, Fernando. 2013. Vitalidad lingüística del mapudungun en Chile y epistemología del hablante. Revista de Lingüística Teórica y Aplicada 51 (1): 53-70.

UnesCo. 1990. Recopilación de materiales didácticos en lengua indígena. Santiago: Unesco.

Viveiros de Castro, Eduardo. 2003. Perspectivismo y multinaturalismo en la América indígena. En Adolfo Chaparro y Christian Schumacher (ed.). Racionalidad y discurso mítico, pp. 191-246. Bogotá: Centro Editorial Universidad del Rosario.

ZúNíIGA, FERNANDO. 2007. Mapudunguwelaymi am? ¿Acaso ya no hablas mapudungún? Acerca del estado actual de la lengua mapuche. Estudios Públicos 105: 9-24. 